\title{
Tecnología del plasma aplicada a problemáticas en el sector hidrocarburos en colombia
}

\author{
Hernán Alfonso Garnica \\ MSc(c) en Física, Universidad Industrial de Santander \\ Profesional, Investigador, Corporación para la Investigación de la \\ Corrosión CIC, Investigador Grupo FITEK, \\ Universidad Industrial de Santander UIS \\ Bucaramanga, Colombia \\ hgarnica@corrosion.uis.edu.co
}

\author{
Ely Dannier Valbuena Niño \\ MSc en Física, Universidad Industrial de Santander \\ Docente Tiempo Completo, Investigador Grupo GINTEP, Uni- \\ versidad Pontificia Bolivariana UPB, Investigador Grupo FITEK, \\ Universidad Industrial de Santander UIS \\ Bucaramanga, Colombia \\ ely.valbuena@upb.edu.co
}

\author{
Valeriy Dugar-Zhabon \\ PhD en Física, Universidad Estatal de Moscú \\ Docente Ttitular, Investigador Grupo FITEK, \\ Universidad Industrial de Santander UIS \\ Bucaramanga, Colombia \\ vdougar@uis.edu.co
}

Resumen-El sector productivo actualmente se ha interesado en el estudio de fenomenologías que involucren mitigar el deterioro por corrosión de los materiales allí empleados, como el desarrollo de nuevos materiales o la aplicación de recubrimientos. En este trabajo se presenta el desarrollo de un nuevo tratamiento (Implantación iónica tridimensional - 3DII) para la protección de superficies metálicas en acero AISI SAE 1010, 1020, 1045 y $316 \mathrm{Ti}$, mediante el empleo de descargas combinadas de arco eléctrico y alto voltaje.

LoS cupones modificados superficialmente son evaluados según técnicas adoptadas por el sector hidrocarburos, tales como los ensayos fisicoquímicos y electroquímicos (permeación de gases $\mathrm{CO} 2$ y H2S). La cuantificación de los daños se llevó a cabo empleando la microscopia confocal 3D y la simulación de esfuerzos por medio del análisis de elementos finitos.

Palabras clave—Corrosión, Implantación, Biocorrosión

Abstract-The phenomenology studies of the measures which can mitigate the corrosion damages of the materials used in the productive sector are now of great interest. The surface coatings and physicochemical modification of the superficial layer make part of these measures. In this work, the AISI SAE 1010, 1020, 1045 y 316 steels are treated in a hybrid discharge: an arc discharge produces high density titanium plasma and a high voltage discharge implants titanium ions.

The corrosion resistance of the treated coupons is evaluated in accordance with the techniques accepted by the hydrocarbon sector, such as the physicochemical and electrochemical tests (CO2 and H2S gas permeability). The quantification of the biocorrosion damages is realized using a 3D confocal microscope and the tensions are simulated by the finite elements technique.

Keywords-Corrosion, Implantation, Biocorrosion.

\section{INTRODUCCIÓN}

La implantación de especies metálicas y no metálicas, tales como el titanio o el nitrógeno, en la superficie del acero al carbono, aún no son empleados en Colombia industrialmente. Actualmente, la protección de dichos materiales se da mediante el uso de recubrimientos no metálicos, transformaciones de micro estructura por cambios térmicos o mediante tratamientos electroquímicos como la carburación y nitruración.

Si nos enmarcamos en la corrosión como una problemáticas del sector hidrocarburo, podemos tener estadísticas palpables y muy específicas, donde los elementos que impactan en la rentabilidad dependen del aumento del tiempo de vida útil de las infraestructuras metálicas, se logrará minimizar los efectos del deterioro por corrosión y por desgaste mecánico.

En Colombia, los costos asociados a los problemas por corrosión en metales se han estimado en casi 1300 millones de dólares al año, lo cual equivale aproximadamente el $1.2 \%$ del Producto Interno Bruto [1], [2]. De estos costos, entre el 30 y el $40 \%$ corresponden a pérdidas generadas por corrosión biológica (bacterias) [3]; con ello la temática cobra un gran interés en el ámbito económico-científico.

Como una alternativa para mitigar los daños producidos por el deterioro de las estructuras a causa de la corrosión, se desarrollan nuevas técnicas de tratamiento superficial mediante métodos plasmicos, las cuales mediante procesos de 
difusión, deposición o implantación proporcionan cambios significativos y mejoras de desempeño en los sustratos metálicos [4-7].

Como una etapa inicial (pre piloto) que permita emplear esta tecnología industrialmente, se propone estudiar la efectividad de la técnica de implantación iónica tridimensional como una alternativa de protección en los aceros [8], [9], para ello se plantea una serie de experimentos que buscan evaluar las superficies modificadas por esta técnica ante los efectos causados por la corrosión en metales de bajo contenido de carbono.

\section{DESARROLLO EXPERIMENTAL}

La base experimental se fundamenta en las normatividades empleadas en la industria del petróleo y gas, generalmente referidas a normas ASTM, NACE y API. La idea es verificar experimentalmente la eficiencia del tratamiento 3DII. En el desarrollo experimental se emplean testigos gravimétricos y biocupones de acero AISI SAE 1010, 1020 y 1045 con bajo contenido de carbono y plugs de acero AISI SAE $316 \mathrm{Ti}$.

La caracterización superficial de los cupones y plugs modificados y no modificados superficialmente se lleva a cabo mediante ensayos gravimétricos y electroquímicos ante corrosión debida a aguas de producción, medida de los defectos asociados a corrosión bacteriana y el mejoramiento de plug de alta presión que son empleados como tapones en líneas de crudo y gas. A continuación se presentan tres etapas para afrontar algunas de las problemáticas presentes en el sector hidrocarburo.

En la primera etapa se realiza un estudio para determinar la velocidad de corrosión de cupones gravimétricos rectangulares planos después de estar inmersos en condiciones estacionarias en una solución de salmuera al 3\%, durante 60 días. Los cupones tienen una geometría de 3" x 3/4" x 1/8" [10] y la superficie presenta un acabado superficial a sandblasting con perfil de anclaje de 0,03 $\mathrm{mm}$. La matriz experimental corresponde a probetas por duplicado en estado de suministro y con implantación de $\mathrm{Ti}$, sin exponer y expuestas en solución de salmuera, en la Fig. 1 se contempla el montaje de los cupones por duplicado.
FIG. 1. MONTAJE DE CUPONES GRAVIMÉTRICOS EN SOLUCIÓN DE SALMUERA

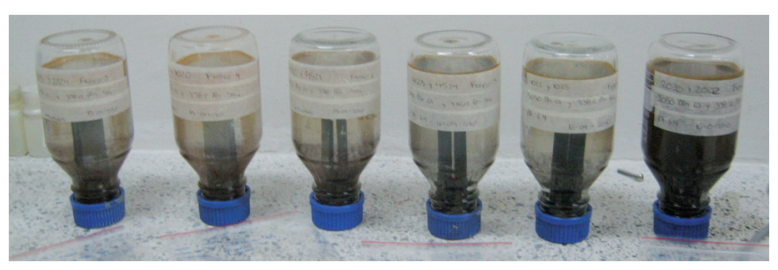

Fuente: Los autores

La segunda etapa busca cuantificar los defectos superficiales en cupones de geometría cilíndrica de espesor $5 \mathrm{~mm}$ y $25,4 \mathrm{~mm}$ de diámetro, con acabado superficial a lija 1200 (rugosidad aproximada de $0,003 \mathrm{~mm}$ ) expuestos a sepas de bacterias sulfato reductoras (BSR). La matriz experimental contempla cupones blancos (sin implantar no inoculados e inoculados bajo ataque bacteriano), cupones implantados con titanio bajo corrosión fisicoquímica (no inoculados) y cupones implantados con titanio inoculados expuestas por un tiempo de 50 días en condiciones estáticas en una salmuera al 0,03\% (esta solución se toma como referencia al agua de producción en petroleras), ver Fig. 2.

FIG. 2. MONTAJE DE BIO CUPONES. (A) CUPÓN NO IMPLANTADO NO INOCULADO A 1 DÍA, (B) NO IMPLANTADO INOCULADO A 50 DÍAS Y (C) IMPLANTADO INOCULADO A 50 DÍAS

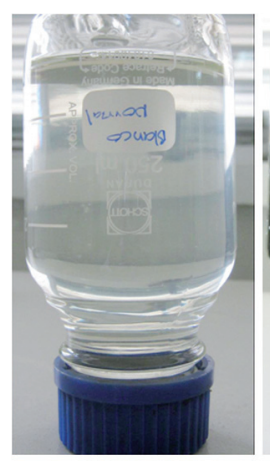

(A)

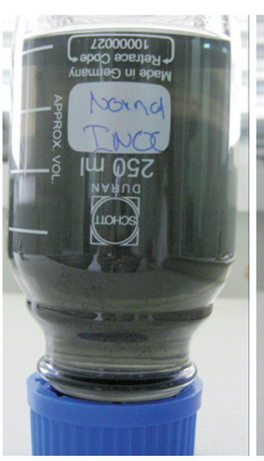

(B)

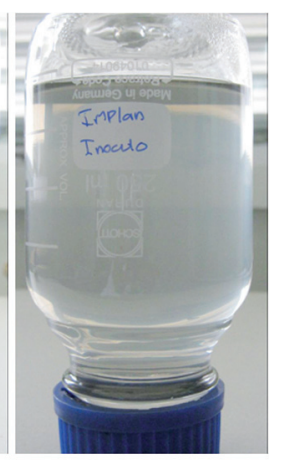

(C)
Fuente: Los autores

En la tercera etapa se realiza el estudio de la problemática referente al daño debido a soldaduras de galling fruto del roce de sus materiales disimiles con presencia de particulado metálico y suciedades entre una unión roscada, presentes en los sistemas retráctiles los cuales utilizan plugs de alta presión para gravimetría (ver Fig. 3), de amplio uso el sector hidrocarburos en la inserción de cupones gravimétricos, probetas eléctricas y sistemas de inyección. El plug de alta presión es modificado superficialmente con iones de titanio mediante la técnica 3DII. 
FIG. 3. (A) SISTEMA RETRÁCTIL (B) PLUG DE ALTA PRESIÓN

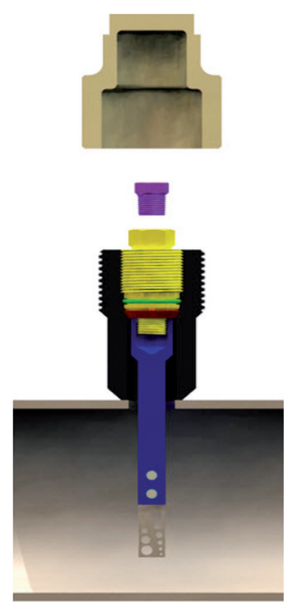

(A)

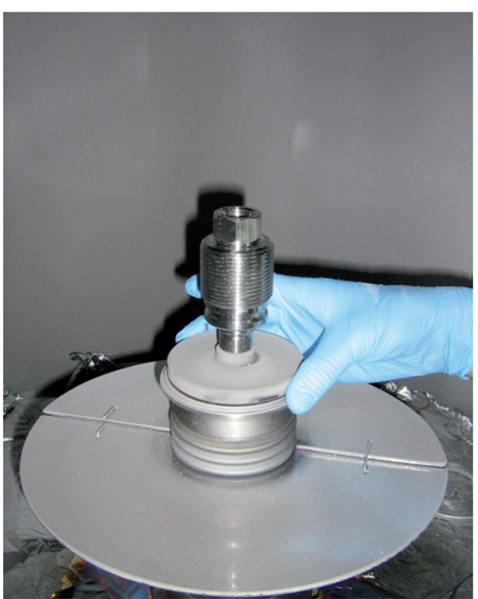

(B)
Fuente: Los autores

\section{RESULTADOS Y DISCUSIÓN}

Los resultados obtenidos por métodos gravimétrico de las velocidades de corrosión en cupones de acero AISI SAE 101, 1020 y 1045 tratado y no tratado superficialmente con iones de titanio fueron calculadas según la normatividad ASTM [11]-[14] y son reportados en la Tabla 1. Los resultados muestran una gran diferencia comprendida entre el $20 \%$ al $110 \%$.

TABLA I

Resultados de las velocidades de corrosión de los ensayos gravimétricos

\begin{tabular}{|c|c|c|}
\hline Material & Implantado & $\begin{array}{c}\text { Vel. Corrosión } \\
\text { (mpy) }\end{array}$ \\
\hline AISI SAE 1010 & N.A. & 1,3 \\
\hline AISI SAE 1020 & N.A. & 0,8 \\
\hline AISI SAE 1045 & N.A. & 0,6 \\
\hline AISI SAE 1010 & Ti & 0,6 \\
\hline AISI SAE 1020 & Ti & 0,6 \\
\hline AISI SAE 1045 & Ti & 0,45 \\
\hline \multicolumn{2}{|c|}{ mpy: milésimas de pulgada por año por año } \\
\hline
\end{tabular}

Fuente: Los autores

Una vez obtenidos los resultados anteriores, se realizó la prueba de ensayos electroquímicas con burbujeo de $\mathrm{CO}_{2}$ para hacer la condición más agresiva, que permitió corroborar la eficiencia de la implantación de Ti como agente mitigador de corrosión, estos cálculos se basaron según la normatividad ASTM [15]-[19] y son reportados en la Tabla 2.
TABLA II

Valores de las velocidades de corrosión obtenidos para los sistemas evaluados

\begin{tabular}{|c|c|c|c|}
\hline Sistema & Implantación & $\begin{array}{c}\text { VelCorr } \\
\text { (mpy) }\end{array}$ & $\begin{array}{c}\text { Eficiencia } \\
\%\end{array}$ \\
\hline 1010 & $\begin{array}{c}\text { Sin } \\
\text { Implantación }\end{array}$ & 22,22 & N.A. \\
\hline 1010 & $\begin{array}{c}\text { Con } \\
\text { implantación } \\
\text { de titanio }\end{array}$ & 1,81 & 91,44 \\
\hline 1020 & $\begin{array}{c}\text { Sin } \\
\text { Implantación }\end{array}$ & 21,06 & N.A. \\
\hline 1020 & $\begin{array}{c}\text { Con } \\
\text { implantación } \\
\text { de titanio }\end{array}$ & 2,65 & 87,53 \\
\hline 1045 & $\begin{array}{c}\text { Sin } \\
\text { Implantación }\end{array}$ & 13,75 & N.A. \\
\hline 1045 & $\begin{array}{c}\text { Con } \\
\text { implantación } \\
\text { de titanio }\end{array}$ & 1,48 & 93,05 \\
\hline
\end{tabular}

Fuente: Los autores

Acorde a lo reportado en la Tabla 2, se observa una disminución mayor al $87,53 \%$ en la velocidad de corrosión en los aceros que fueron implantados con Ti en comparación con los aceros del mismo tipo sin implantación.

En la segunda etapa se analizaron las superficies de los cupones implantados y no implantados después de estar inmersos en la solución con BSR. Las superficies fueron caracterizadas ópticamente con ayuda de un microscopio de alta resolución de campo brillante automatizado imagen 01M ZIISS. En la Fig. 4 (A) se observan picados entre 15-30 micras a profundidades de 8-15 micras en un área analizada de 284 × 212 micras. En la Fig. 4 (B) se evidencia picado con diámetros de 2 micras a profundidades de 3 micras en un área analizada de 142 × 106 micras.

FIG. 4. IMÁGENES DE LOS DEFECTOS EN ESCALA DE GRISIS

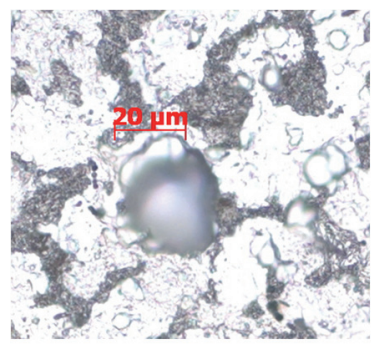

(A)

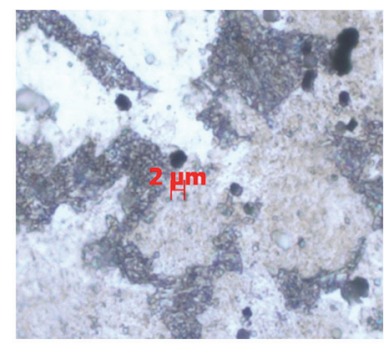

(B)
Fuente: GOTS - UIS

Por último se presenta el proceso estructurado desde el diseño gráfico dimensional, la simulación de esfuerzos mediante el análisis finito como 
se muestra en la Fig. 5, se pasó por la fabricación, modificación superficial con $\mathrm{Ti}$, hasta terminar con la evaluación y comparación de las superficies implantadas y no implantadas en un banco de pruebas de repetitividad mecánica.

FIG. 5. DISTRIBUCIÓN DE ESFUERZOS EN LOS FILETES DE ROSCAS EN PLUG (A) SIN TRATAMIENTO Y (B) CON TRATAMIENTO

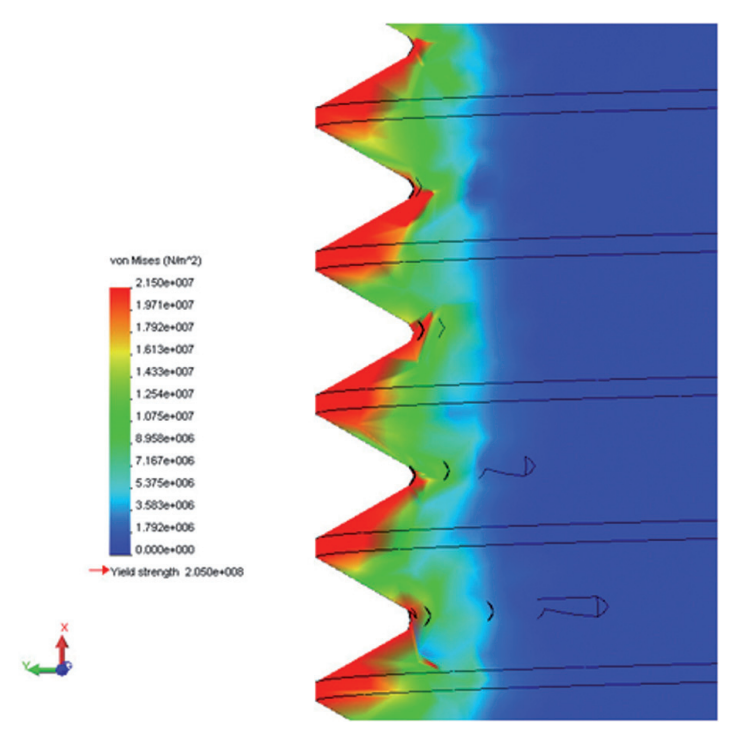

(A)

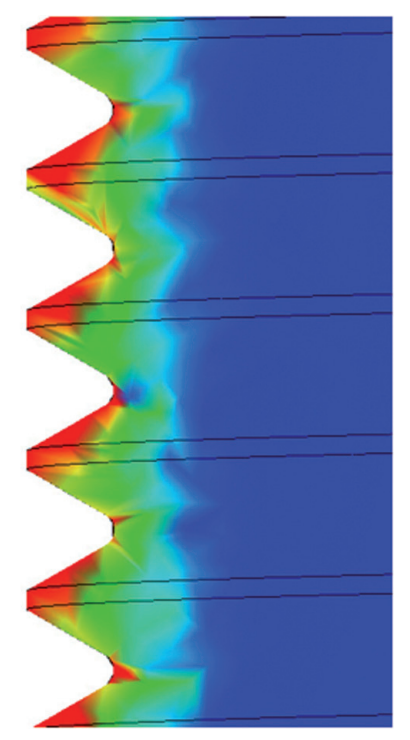

(B)

\section{CONCLUSIONES}

El tratamiento superficial de los aceros AISI SAE 1010,1020 y 1045 mediante la implantación iónica tridimensional de iones de titanio realizada en el reactor MOSMET [20], resulta un mejoramiento significativo de resistencia a la corrosión fisicoquímica y se evidencia que el proceso es eficiente.

De acuerdo a los resultados obtenidos mediante técnicas electroquímicas, se observa una disminución en la velocidad de corrosión mayor al 87,53\% para los cupones implantados con Ti, lo cual indica que el proceso que la técnica empleada genera una disminución en la tendencia general que tiene el hierro de buscar la forma más estable o de menor energía interna.

El experimento muestra que la técnica 3DII es eficiente en la reducción del daño superficial producido por las BSR, cuantificándose picaduras del orden del $0,003 \mathrm{~mm}$, con respecto a las probetas no implantados y sometidas al ataque bacteriano, las cuales reportan picaduras del orden de los $0,03 \mathrm{~mm}$. Se puede concluir que la implantación de iones de titanio minimiza la acción dañina de los microorganismos sulfatos reductores.

En los plugs de alta presión se logró mejorar el factor de diseño en un 16\%, con mejoras del 15\% de desempeño en pruebas de presión los plugs implantados con $\mathrm{Ti}$ y ante las pruebas de repetitividad mecánica presentó un aumento en la vida útil del 90\%.

\section{AGRADECIMIENTOS}

Los autores presentan sus agradecimientos al grupo de biocorrosión, fisicoquímica y taller de mecánica fina de la corporación para la investigación de la corrosión - CIC; al grupo de física y tecnología del plasma de la universidad industrial de Santander y su proyecto MOSMET financiado por Colciencias, al grupo de óptica y tratamiento de señales en especial al PhD. Arturo Plata G. y al PhD. Piotr Tsygankov profesor asociado al departamento de ingeniería del plasma de la universidad estatal de Bauman Moscú.

\section{REFERENCIAS}

[1] C. Arroyave, et. al. (1997). "Corrosion problems and their incidence on Colombian Economy". Proc. 10th Asia Pacific Corrosion Control Conference. Bali, Indonesia, 27-31 October. Paper 12.1/6. T.S. Rao, A.J. Kora, B. Anupkumar, S.V. Narasimhan, R. Feser. "Pitting corrosion of titanium by a freshwater strain of suplase redu- 
cing bacteria (Desulfovidrio vulgaris)". Corrosion Science, 47 (2005) 1071-1084.

[2] M. B. Kermany and D. Harrop, BP Internacional, SPE 29784, 1995

[3] Srivastava, V., 1992).

[4] Vladimir I. Khvesyuk, Piotr A. Tsygankov, The use of a high-voltage discharge at low pressure for 3D ion implantation, Surface and Coatings Technology 96, (1997) 68-74.

[5] A. S. Pokrovskaya-Saboleva and B. N. Klarfeld, Russian J. Appl, Phys., 32, 993 (1957).

[6] McClure G.W., High voltage glow discharge in D2 gas. Diagnostic measurements, Phys. Rev. 124 (4), (1961) 969-982.

[7] V.D. Dugar-Zhabon, J. Dulce Moreno, P.A. Tsygankov, High voltage pulse discharge for ion treatment of metals, Rev. Sci. Instrum. 73, (2002) 828-830.

[8] Dulce Moreno, H. Jaime. Implantación Iónica Tridimensional Mediante Descargas de Alto Voltaje a Bajas Presiones del Dispositivo Júpiter. Bucaramanga, 2002, 204 h. Trabajo de grado (Doctor en Ciencias Naturales - Física). Universidad Industrial de Santander. Facultad de Ciencias. Escuela de Física.

[9] V.D. Dugar-Zhabon, J. Castro Blanco, H.J. Dulce Moreno, P.A. Tsygankov, Device "Jupiter" for ion implantation, Rev. Col. Fís. Vol. 31 No. 2, (1999) 181-184.

[10] E. D. V. Niño, H. Garnica, V. Dugar-Zhabona, D. Martínez, F. Durán “Caracterización de cupones gravimétricos de acero API 5LX 65 implantado por iones de nitrógeno e iones de titanio", Rev. Col. Fís. 43 (2011) 134-137.

[11] NACE RP 0775-05 Preparation, Installation, Analysis and Interpretation of Corrosion Coupons in Oilfield Operations.

[12] ASTM G1-03 Standard Practice for Preparing, Cleaning and Evaluating Corrosion Test Specimens.

[13] ASTM G31-04 Practice for Laboratory Immersion Corrosion testing of Metales.

[14] ASTM G59-97 Standard Test Method for Conducting Potentiodynamic Polarización Resistance Measurements.
[15] ASTM G3-99 Standard Practice for Conventions Applicable to Electrochemical Measurements in Corrosion Testing.

[16] ASTM G5-99 Standard Reference Test Method for Making Potentiostatic and Potentiodynamic Anodic Polarization Measurements.

[17] ASTM G102-99 Standard Practice for Calculation of Corrosion Rates and Related Information from Electrochemical Measurements.

[18] NACE RP 0775-05 Preparation, Installation, Analysis and Interpretation of Corrosion Coupons in Oilfield Operations.

[19] ASTM G185 - 06 Standard Practice for Evaluating and Qualifying Oil Field and Refinery Corrosion Inhibitors Using the Rotating Cylinder Electrode.

[20] Proyecto Colciencias número 321 de 2005 Estudio de un Nuevo Método de Modificación de Superficies de Metales - MOSMET, Valeriy DugarZhabon. 